\title{
Optimal dispatch of hybrid energy islanded microgrid considering V2G under TOU tariffs
}

\author{
Yiwei Ma ${ }^{1,}$, Yuyang Chen ${ }^{1}$, Xin $\mathrm{Chen}^{2}$, Fuchun Deng ${ }^{1}$, and Xiantong Song ${ }^{1}$ \\ ${ }^{1}$ School of Automation, Chongqing University of Posts and Telecommunications, Chongqing 400065, P. R. China \\ ${ }^{2}$ Smart Energy Technology Research Centre, Chongqing 400065, P. R. China
}

\begin{abstract}
In order to achieve a prospective economic effect of renewable energy generations and vehicle to grid (V2G), this paper proposes an optimal dispatch method of wind-PV-battery microgrid considering V2G under time-of-use (TOU) tariffs for those isolated communities in remote islands and mountainous areas. A cooperative dispatch strategy and an optimal dispatch model are both presented for the total operation cost minimization and higher utilization of renewable energy generation. Finally, the simulation results show that the proposed method is effective and feasible.
\end{abstract}

\section{Introduction}

In recent years, the environmental problems caused by fossil energy are becoming more serious, as it will produce a large amount of harmful gas such as carbon dioxide, sulfur dioxide and other emissions to cause a series of environmental pollution in the process of producing and combustion. This drives the green renewable energy generations (such as wind turbine (WT), solar photovoltaic (PV), etc.), microgrid (MG) and other advanced energy technologies research and development [1]. It is well known that microgrids is defined as the effective and feasible solution to the outstanding grid-connected problem of the intermittent renewable energy generations like WT and PV, thus microgrids have received considerable attentions by domestic and foreign researchers. At present, the economic dispatch is a very important and outstanding problem of this kind of new energy power system.

At present, there are a lot of researches on microgrid economic dispatch modeling, optimization and simulation in the literatures [2-4]. Literature [5] introduces the situation of thermal power generation such as diesel generator and environmental pollution emissions, and proposes an economic dispatch method by allocating various distributed generation (DG) for the minimal operation cost of microgrid. Similarly, Literature [6] gives a V2G economic dispatch method as to allocate battery energy storage (BS), electric vehicle (EV) and other DGs as to minimize the total operating cost of microgrid. Literature [7] presents a economic dispatch model of the minimum economic operating cost under TOU tariffs. Literature [8] analyses the shortcomings of microgrid coordinated operation, and gives an optimal economic operation strategy according to the charging and discharging characteristics of BS and EVs and TOU tariffs. Furthermore, a multiple optimal dispatch model is presented for microgrid energy management considering TOU tariffs in Literature [9]. In addition, based on the theory of price elasticity of electric demand, Literature [10] presents a flexible load dispatching model of active distribution network with time-of-use price mechanism and a flexible load control strategy. Literature [11] makes an analysis of the influence of TOU price on the charging probability of EVs, and then gives an optimal dispatch strategy obtained by using PSO algorithm. Literature [12] presents an optimal operation model for wind-PV-battery household user-side microgrid based on demand response and TOU tariffs. In summary, these studies have paid more attentions to dispatch economic and environmental aspects, but less for the coordination dispatch strategy among various microgrid DG units and a special flexible load of EV under demand response mechanism.

Therefore, this paper presents an optimal dispatch method as to minimize the total economic cost for a wind-PV-battery hybrid energy microgrid considering V2G under TOU tariffs. Fig. 1 shows the detailed system framework of hybrid renewable energy islanded microgrid and a special source-load unit of EV, which can access to grid by the static switch. The distributed units of PV, WT, BS and EV and load are respectively connected to the bus line by its own feeder line. Thus, EV can buy from or sell to the external grid, and also charge from or discharge to microgrid by the load controller (LC). Therefore, the coordinated dispatch strategy among these distributed generations and loads under TOU tariffs is presented to make full use of renewable energy firstly. And the economic dispatch model considering V2G and demand response is given to minimize the system operation cost. Finally, the effectiveness and effectiveness of the presented method were verified by simulation results, which show the proposed method can achieve the minimum system operation cost and the maximum renewable energy utilization.

\footnotetext{
* Corresponding author: mayw@cqupt.edu.cn
} 


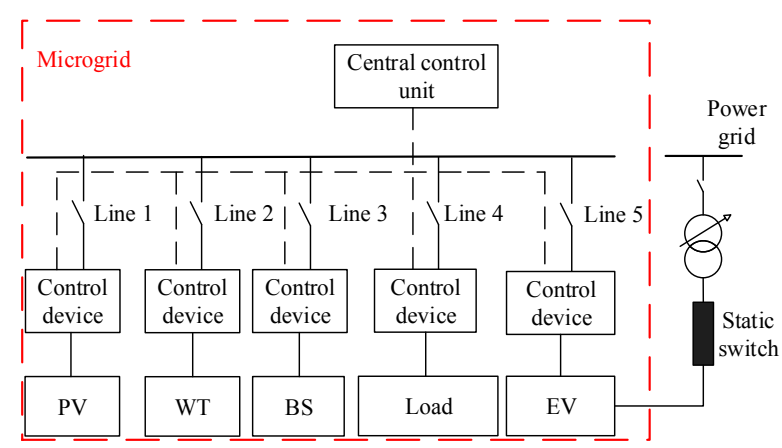

Fig. 1. Diagram of Wind-PV-BS-EV microgrid.

\section{Coordination dispatching strategy}

With the application of a large number of EVs, the microgrid with charging and discharging devices of EVs is becoming more and more popular. Therefore, it is especially important to study the dispatching model of EV connecting to microgrid. At present, the technology of $\mathrm{V} 2 \mathrm{G}$ is widely used to complete the connection between EV and grid. Considering the frequent charging of electric vehicles will affect the battery life, the EVs can be centrally charged and discharged according to the change of load and the mechanism of TOU tariffs. As shown in Fig. 2, it can be divided into the following stages: I . At night, the load is in a low valley period, and the electricity price is low. the EV can centrally charge to absorb the night-time wind power resources. II. In the daytime, the excess power of the EV can be transmitted to the power grid in order to enhance the power supply stability, when the load is in the peak, and the electricity price is higher.III. The EV is generally in a charging or holding state when the load is in a flat section.

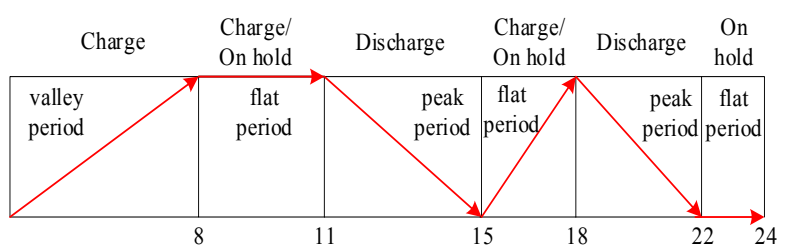

Fig. 2. Charge/discharge mode in order of electric vehicle.

According to electricity consumption, 24 hours of a day will be divided into peak, valley and flat stage, as follows: Peak hours (11:00-16:00,19:00-22:00); Flat hours (08:00-11:00,16:00-19:00,22:00-24:00); Valley hours (00:00- 08:00). The dispatching time of operation of microgrid is set to one hour. Real-time monitoring of PV, WT and load demand during scheduling time, as well as the charging status of the battery. According to the state of charge (SOC) of the battery at different time periods of the current dispatching time, then the EV is allowed to participate in the dispatching. At the same time, the optimal goal is to minimize the system operation cost during the dispatching period.

In the scheduling strategy, $\Delta P_{t}$ is the net load of $\operatorname{MG}\left(\Delta P_{t}=P_{L}-P_{W T}-P_{P V}, P_{L}\right.$ is total load demand, $P_{W T}$ is WT output power, $P_{P V}$ is PV output power), $S O C$ is
BS remaining capacity, $S O C_{\min }$ is minimum capacity for battery operation, $P_{d c h \text { max }}, P_{c h \text { max }}$ are the battery maximum discharge power and maximum charging power, $P_{R E}$ is total power output of WT and PV. $t$ is a scheduling time of one day, one day is divided into 24 scheduling periods, and one hour is seen as a scheduling time.

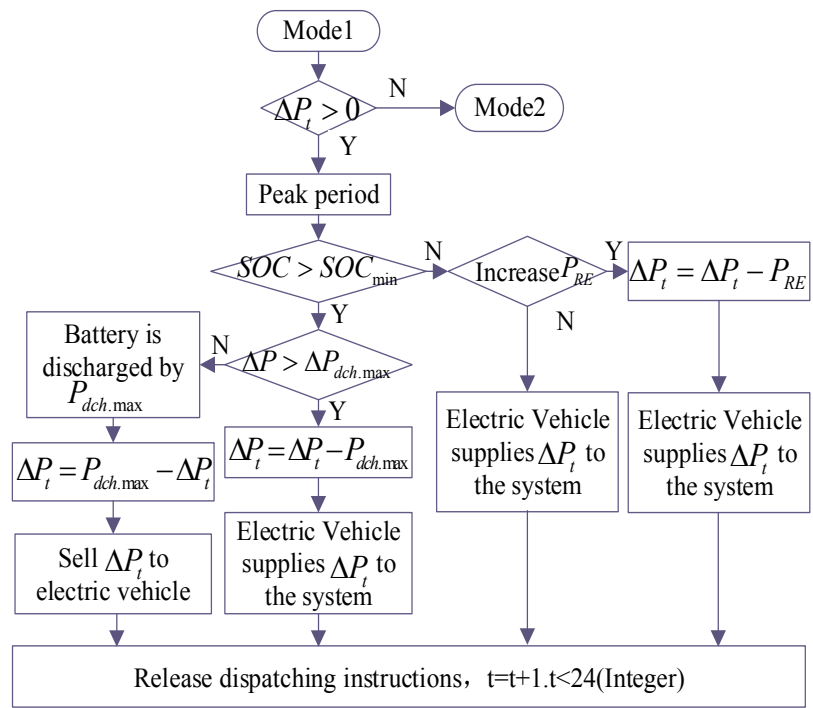

Fig. 3. Strategy for the insufficient renewable energy power (Peak period).

Fig. 3 is the scheduling strategy for the peak time period when WT and PV output power is less than load demand, that is, when the net load of the system is less than 0 due to the shortage of WT and PV and peak electricity consumption. At this point, the cost of purchasing price is higher, so choose the priority battery to supply power, and then judge whether the battery reaches the minimum allowable operation capacity.If it is not achieved, consider whether to increase WT and PV capacity $P_{R E}$, and then purchase electricity from EVs to supply $\mathrm{MG}$; if the requirements are met, the battery supplies power to MG, and when the maximum discharge power of battery $P_{d c h \text { max }}$ is greater than $\Delta P_{t}$, Selling electricity to EVs on the basis of meeting the power supply of the grid, if the maximum discharge power of battery $P_{d c h \text { max }}$ is less than $\Delta P_{t}$, The battery is first supplied until it reaches its minimum capacity, and then the remaining required power is supplied by the EV.

Fig. 4 is the scheduling strategy for the valley period when WT and PV is less than the user's load demand. In this dispatching strategy, the microgrid is in a flat valley period under the condition of insufficient wind and PV power supply, giving priority to purchasing electricity from EVs with low electricity prices to make up for the lack of power supply to the system, then judging battery capacity, when the battery capacity does not reach the maximum capacity $S O C_{\max }$, the EV should be used to charge BS as much as possible, which is used to prepare for the electric output during peak-time. 


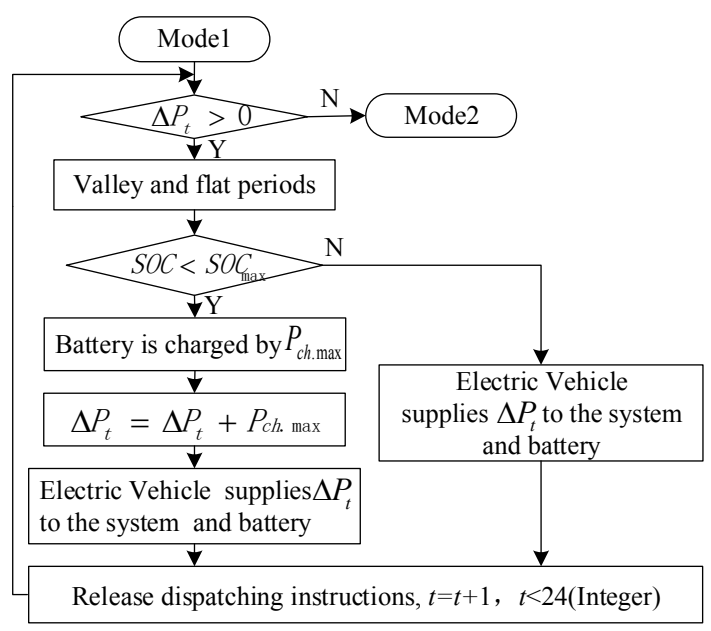

Fig. 4. Strategy for the insufficient renewable energy power (Valley and flat periods).

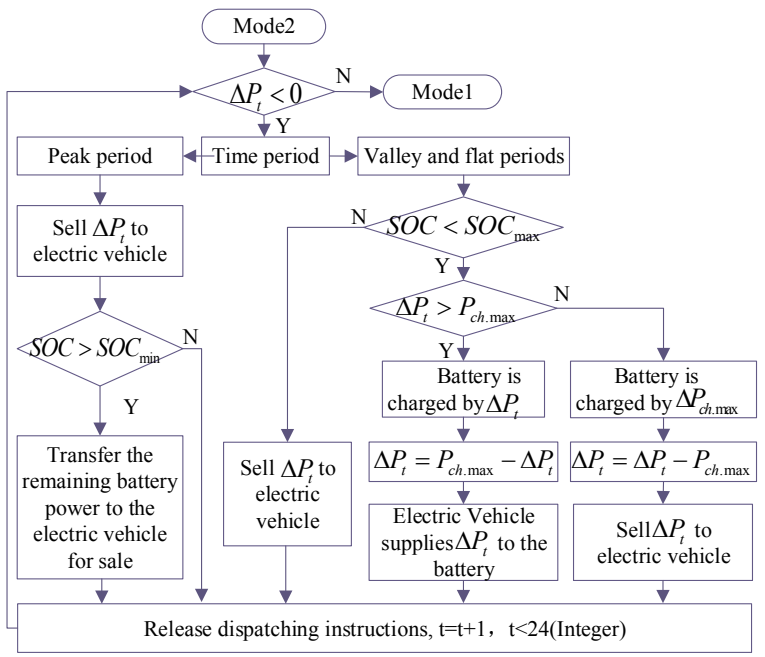

Fig. 5. Strategy for the sufficient renewable energy power.

Fig. 5 is the scheduling strategy for situations where WT and PV is greater than load demand.In this strategy, when load is in peak-tme, the $\Delta P_{t}$ will be sold to EVs first. It is further determined whether the remaining battery capacity $S O C$ is greater than the minimum capacity $S O C_{\min }$, if so, the remaining capacity of $\mathrm{BS}$ is also transferred to the $\mathrm{EV}$ for sale, which is used to reduce the electricity cost. When load is in flat valley period, the $\Delta P_{t}$ is preferably transferred to the battery for charging until the maximum capacity of battery is reached, if there is any surplus, transfer the excess power to $\mathrm{EV}$ for sale, if not enough, EV charging battery until the maximum capacity of the battery is reached.

\section{Optimization dispatching model}

\subsection{The optimization objective}

Total scheduling cost objective function:

$$
\min C_{M G}\left(C_{M G}(P)\right)=\sum_{t=1}^{K} \sum_{i=1}^{N} C_{i}\left(P_{i}(t)\right)+C_{s}
$$

$$
C_{i}\left(P_{i}(t)\right)=C_{R E . i}\left(P_{i}(t)\right)+C_{B}\left(P_{B}(t)\right)+C_{E V}
$$

Where $C_{M G}$ is the cost of power $P$ generated by $\mathrm{MG}$ in dispatching period $K t ; C_{i}\left(P_{i}(t)\right)$ is the total cost of the i-th DG output power $P_{i}(t) ; K$ is the number of optimized period; $N$ is the number of DG; $P_{i}(t)$ is the $i$-th DG output; $C_{s}$ is TOU purchase cost; $C_{R E . i}\left(P_{i}(t)\right)$ is the cost of renewable energy generation; $C_{B}\left(P_{B}(t)\right)$ is the cost of battery discharge, $C_{E V}$ is the cost of EV power supply.

(1)Renewable energy generation cost:

$$
\begin{aligned}
& C_{R E . i}\left(P_{i}(t)\right)=C_{D C . i}\left(P_{i}(t)\right)+C_{M O . i}\left(P_{i}(t)\right) \\
& -M_{E S . i}\left(P_{i}(t)\right)
\end{aligned}
$$

Where $C_{D C . i}, C_{M O . i}$ and $M_{E S . i}$ is the depreciation expense of the i-th renewable DG output power $P_{i}(t)$, the operating maintenance costs and subsidies.

(2) Battery energy storage system operating cost:

$$
\left\{\begin{array}{l}
C_{B}\left(P_{B}(t)\right)=\gamma_{B} \times P_{B . d c h}(t) \\
\gamma_{B}=\frac{C_{i . B}+C_{M O . B}}{E_{a . B}}
\end{array}\right.
$$

Where $C_{B}\left(P_{B}(t)\right)$ is the battery power generation costs when the discharge power is $P_{B}(t) ; P_{B . d c h}(t)$ is the discharge power; $\gamma_{B}$ is power generation cost coefficient, which is the ratio of the total cost of battery purchase and maintenance to the total discharge during the life cycle.

(3) Electric vehicle power supply cost:

$$
C_{E V}=\sum_{t=1}^{T}\left(C_{\text {dis.t }} P_{\text {dis.t }}\right)+C_{k}
$$

Where $C_{d i s . t}$ is $\mathrm{EV}$ discharge price during the t-th period; $P_{\text {dis.t }}$ is the EV discharge power during the t-th period; $C_{k}$ is the cost of EV maintenance.

\subsection{Constraint conditions}

(1) Constraints on the balance of power supply and demand:

$$
\sum_{i=1}^{G} P_{i}(t)=P_{e}(t)+P_{T}(t)
$$

Where $P_{i}(t)$ is the output power of type i DG supply in ttime; $P_{e}(t)$ is the power beyond the load requirements of the microgrid; $P_{T}(t)$ is the total system load demand.

(2) Constraints on the renewable energy output:

$$
P_{i \text { min }} \leq P_{i} \leq P_{i \text { max }}
$$

Where $P_{i . \min }$ is the minimum output power; $P_{i \cdot \max }$ is the maximum output power; $P_{i}$ is the output power of renewable energy.

(3) Constraints on the start and stop time of WT:

$$
T_{W T . i} \geq T_{W T \text {.min. } i}
$$

Where $T_{W T . i}$ is the start-stop time of the i-th WT; $T_{W T \text {.min.i }}$ is the shortest start-stop time of the i-th WT.

(4) Constraints on the capacity of BS:

$$
S O C_{\min } \leq S O C(t) \leq S O C_{\max }
$$

Where $S O C_{\min }$ is the minimum capacity; $S O C(t)$ is BS capacity at t-time; $S O C_{\max }$ is the maximum capacity.

(5) Constraints on the power of electric vehicle: 


$$
\begin{aligned}
P_{c h . t \text { min }} & \leq P_{c h}(t) \leq P_{\text {ch.t.max }} \\
P_{d c h . t \text { min }} & \leq P_{d c h}(t) \leq P_{d c h . t \text { max }}
\end{aligned}
$$

Where $P_{\text {ch.t.min }}$ is the minimum charging power of EVs; $P_{\text {ch.t. max }}$ is the maximum charging power of EVs; $P_{d c h . t \text { min }}$ is the minimum discharge power of EVs; $P_{d c h . t \text { max }}$ is the maximum discharge power of EVs.

\section{The case analysis}

\subsection{The basic data}

The system parameters of this paper are shown in Table 1 and Table 2.

Table 1. WT and PV parameters

\begin{tabular}{ccc}
\hline & WT & PV \\
\hline Number & 2 & 2 \\
Rated power & $200 \mathrm{~kW}$ & $100 \mathrm{~kW}$ \\
Rated voltage & $380 \mathrm{~V}$ & $380 \mathrm{~V}$ \\
\hline
\end{tabular}

Table 2. Battery and load parameters

\begin{tabular}{lc|cc}
\hline \multicolumn{2}{c|}{ BS } & \multicolumn{2}{c}{ Load parameter } \\
\hline Capacity & $600 \mathrm{~kW}^{*} 6 \mathrm{~h}$ & EV & $300 \mathrm{kWh}$ \\
Inverter power & $600 \mathrm{~kW}$ & Peak load (Load) & $250 \mathrm{~kW}$ \\
\hline
\end{tabular}

In this paper, $24 \mathrm{~h}$ is the optimal calculation period, the data of the user's load, power of the wind turbine and photovoltaic system are shown in Fig. 6, Fig. 7 and Fig. 8 over different time periods within $24 \mathrm{~h}$.

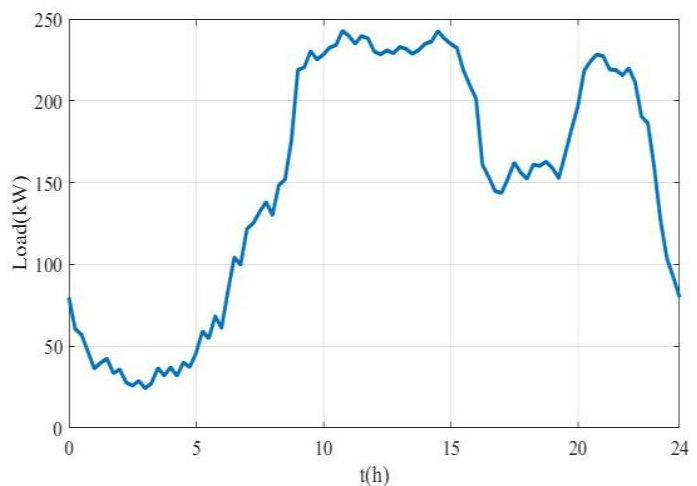

Fig. 6. Profile of system load.

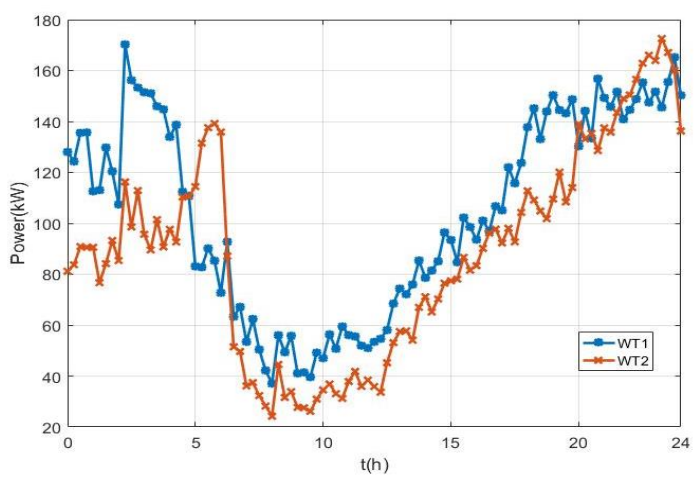

Fig. 7. Full-day output curve of WT.

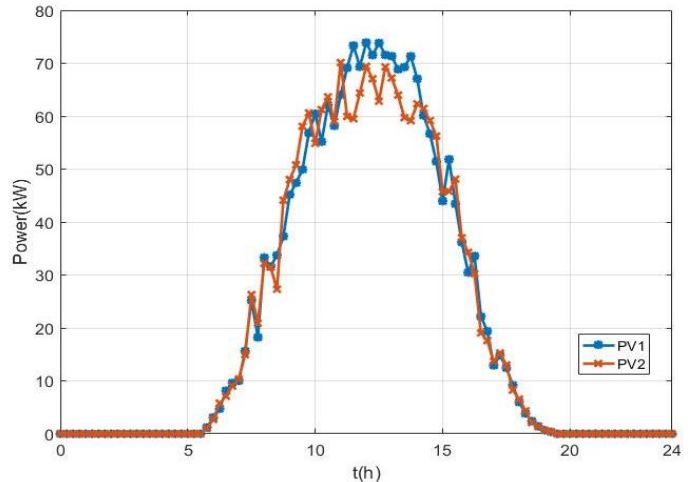

Fig. 8. Full-day output curve of PV.

As can be seen from Fig. 6, 10h-15h and 19h-22h are peak periods of load demand, and $0 \mathrm{~h}-6 \mathrm{~h}$ is valley period. And Fig. 7 shows that WT output is very small during the daytime, but higher at night, and it can reach the maximum value. Fig. 8 shows that PV can convert solar energy into more electricity during $6 \mathrm{~h}-18 \mathrm{~h}$, which can be used to meet the load demand of the daytime system.

\subsection{Simulation results}

The specific TOU tariffs are shown in Table 3.

Table 3. Time-of-Use

\begin{tabular}{ccc}
\hline \multirow{2}{*}{ Classify } & \multicolumn{2}{c}{ Price $(\mathrm{RMB} / \mathrm{kW} * \mathrm{~h})$} \\
\cline { 2 - 3 } & Purchase & Sale \\
\hline Valley time (0h-6h) & 0.3 & 0.2 \\
Flat time (6h-10h,13h-17h,22h-24h) & 0.6 & 0.4 \\
Peak time (10h-13h,17h-22h) & 0.9 & 0.6 \\
\hline
\end{tabular}

Table 3 shows that the lowest electricity price in the daily $0 \mathrm{~h}-6 \mathrm{~h}$, and users can choose to charge the battery with EVs during this period. The highest electricity prices in $10 \mathrm{~h}-13 \mathrm{~h}$ and $17 \mathrm{~h}-22 \mathrm{~h}$, and microgrid is preferred to EVs if there is excess power. In the remaining time period, it is considered to purchase electricity or sell electricity to EVs according to specific conditions.

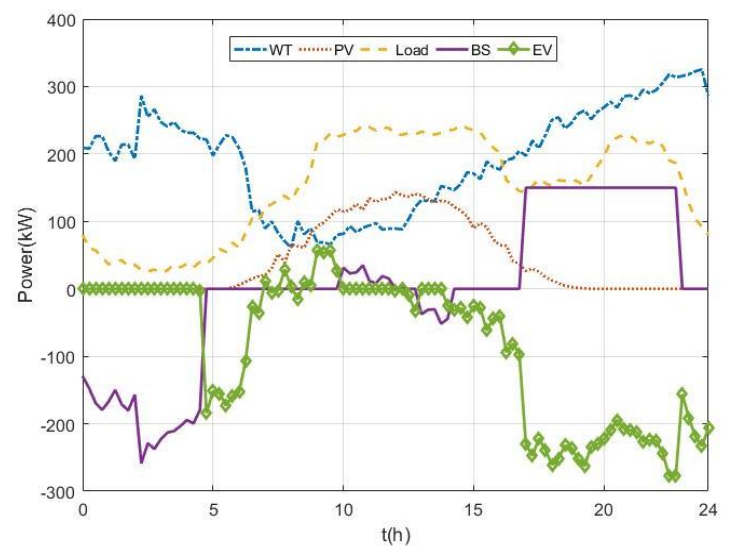

Fig. 9. Optimized scheduling profile with EV. 


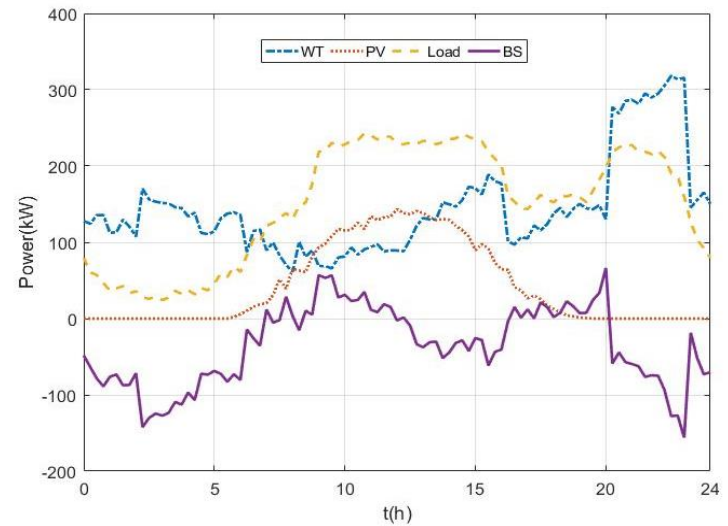

Fig. 10. Optimized scheduling profile without EV.

The scheduling results are shown in Fig. 9 and Fig. 10. Fig. 9 indicates the optimized scheduling profile with EV, Fig. 10 indicates the optimized scheduling profile without EV. Compared with Fig. 9 and Fig. 10, it can be seen that when EVs are connected to MG: during 0:00-5:00, the output of WT is much higher than load and there is a lower electricity price, on the basis of satisfying load demand, transferring excess electricity to BS until it reaches its maximum capacity; during 5:00-10:00, the EV is used in peak shaving to smooth the net load fluctuation; during 10:00-12:30, WT and PV is not enough to meet the load demand, and because of the high electricity price, BS is used for the system to supply power and replenish the insufficient power; during 12:30-13:00, WT and PV is sufficient and there is a higher electricity price, and the excess electricity is transferred to EVs, which are then sold through it; during 13:00-24:00, WT and PV is sufficient, when the electricity price is lower, the excess power of the system is transferred first to charge BS, and when the electricity price is higher, the excess power and the battery power are transferred to the EV for sale. When the EVs is not connected to the microgrid: BS is used for peak shaving and net load fluctuation of the system all day long. During 0:00-6:30, due to the excessive WT and the system could not consume it, so consider shutting down WT1; similarly, at 16:30-20:15, 23:00-24:00, WT2 can be turned off to reduce costs, but it will reduce renewable energy utilization.

Table 4. Comparison of the two cases

\begin{tabular}{ccc}
\hline & Connected EV & Non-connected EV \\
\hline Cost (RMB) & 3286.4 & 3915.2 \\
\hline $\begin{array}{c}\text { Renewable energy } \\
\text { utilization rate (\%) }\end{array}$ & 100 & 90.6 \\
\hline Cost savings (RMB) & \multicolumn{3}{c}{628.8} \\
\hline
\end{tabular}

Conclusions can be drawn from Table 4: When the EV is connected to the microgrid, the operation cost of the microgrid is RMB3286.4 because of the large-scale electricity sale behavior of EV after $13 \mathrm{~h}$; when EV is not connected to the microgrid, because it is impossible to get more benefits by selling electricity, it can only adjust the operation status of each module to reduce the cost, such as shutting down a WT when there is plenty of wind power. At this time, the operating cost of the microgrid is RMB3915.2. Compared with the two cases, the cost is reduced by RMB628.8 when considering the connection of EV to the microgrid. and when connected to EVs, renewable energy utilization rate is $100 \%$, compared with $90.6 \%$ when not connected. It can be seen that the cost of connecting EVs to microgrid under TOU tariffs is obviously lower and more environmentally friendly.

\section{Conclusion}

Microgrid and V2G both can provide prospective economic effects to renewable energy power system and power consumers. In view of the characteristics of movable energy storage device for electric vehicle, it can charge from external grid or microgrid to absorb the excess renewable energy power, and discharge to microgrid to compensate the electric power. this paper presents a novel optimal dispatch scheme based on the proposed coordination dispatch strategy among microgrid distributed generation units such as WT, PV, BS and EV and the dispatch optimization model under TOU tariffs, which can achieve the minimum operation cost of microgrid. The simulation results show the proposed method can reduce the electric power cost and enhance the renewable energy absorptions.

This research was supported by the National Natural Science Foundation of China (No.61703068 \& No.61673079), and the Science and Technology Research Program of Chongqing Municipal Education Commission (No. KJ1704097).

\section{References}

1. Ma Yiwei, Yang Ping, Wang Yuewu, et al. Typical characteristics and key technologies of microgrid, Automation of Electric Power Systems, 39, 168-175 (2015)

2. Sundari R, Stephen B B, Tyler J. Reliability optimization in the islanded mode of microgrid, Energy and Power Engineering, 11,103-114 (2017)

3. Dai Rui, Wang Jinmei, Huo Da, et al. Research on microgrid control strategy, Information and Communication, 1, 24-26 (2017)

4. Ma Yiwei. Optimal sizing and coordinated control of distributed generation units in islanded microgrid, Guang Dong: South China University of Technology (2015)

5. Galia M, Vassil G. Energy Scheduling for island microgrid applications, Communications and Computers, 6, 281-290 (2016)

6. Ke Qijiang, Liu Xiao, Liu Bo. Micro-grid economic dispatching of containing multiple distributed generations, Ningxia Electric Power, 3, 7-12 (2017)

7. Nisha K. An overview of bidirectional DC-DC converter topologies and control strategies for interfacing energy storage systems in microgrids, Electric Engineering, 6, 11-17 (2018).

8. Ricardo L VA, Dorel S R, Tesoro Elena D C H. Overvoltage condition assessment of a microgrid due 
to faults occurring in the islanded mode of operation, Energy and Power Engineering, 7, 525-534 (2015)

9. Ross M, Abbey C, Bouffard F. Multiobjective optimization dispatch for microgrids with a high penetration of renewable generation, IEEE Transactions on Sustainable Energy, 6, 1306-1314(2015)

10. Sun Jianjun, Zhang Shize, Zeng Mengdi, et al. Multiobjective optimal control for flexible load in active distribution network considering time-of-use tariff,
Transactions of China Electrotechnical Society, 33, 401-412(2018)

11. Xu Congqi, Gao Ruilin, Jia Guizhi, et al. Scheduling of microgrid considering electrical vehicles of timeof-use tariffs, Journal of Electric Engineering, 12,1220(2017)

12. Huang Wei, Ma Xiyuan, Huo Jinyong, et al. Optimal operation of household user-side microgrid considering time-of-use price and demand response, Southern Power System Technology, 9, 47-53(2015) 